\title{
The Molecular Signature of High-intensity Training in the Human Body
}

\section{다 (i) $($ 웅}

Authors

Patrick Wahl1, 2, 3, Wilhelm Bloch ${ }^{2,4}$, Sebastian Proschinger ${ }^{5}$

\section{Affiliations}

1 Institute of Cardiovascular Research and Sport Medicine, German Sport University Cologne, Cologne, Germany

2 The German Research Center of Elite Sport Cologne, German Sport University Cologne, Koln, Germany

3 MSH Medical School Hamburg, Institute of Interdisciplinary Exercise Science and Sports Medicine, Hamburg, Germany

4 Molecular and Cellular Sport Medicine, German Sport university, Cologne, Germany

5 Department for Molecular and Cellular Sports Medicine, Institute for Cardiovascular Research and Sports Medicine, German Sport University Cologne, Cologne, Germany

Key words

haemodynamic stress, calcium signalling, metabolic stimuli, skeletal muscle, immune system, cardiovascular system

accepted 13.07.2021

published online 15.07 .2021

\section{Bibliography}

Int J Sports Med 2022; 43: 195-205

DOI 10.1055/a-1551-9294

ISSN $0172-4622$

(c) 2021. The Author(s).

This is an open access article published by Thieme under the terms of the Creative Commons Attribution-NonDerivative-NonCommercial-License, permitting copying and reproduction so long as the original work is given appropriate credit. Contents may not be used for commecial purposes, or adapted, remixed, transformed or built upon. (https://creativecommons. org/licenses/by-nc-nd/4.0/)

Georg Thieme Verlag KG, Rüdigerstraße 14,

70469 Stuttgart, Germany

\section{Correspondence}

Prof. Wilhelm Bloch

German Sport University, Molecular and Cellular Sport

Medicine,

Carl-Diem-Weg 6

50933 Cologne

Germany

Tel.: 004922149825380, Fax : 004922149828370

W.Bloch@dshs-koeln.de

\section{ABSTRACT}

High-intensity training is becoming increasingly popular outside of elite sport for health prevention and rehabilitation. This expanded application of high-intensity training in different populations requires a deeper understanding of its molecular signature in the human body. Therefore, in this integrative review, cellular and systemic molecular responses to high-intensity training are described for skeletal muscle, cardiovascular system, and the immune system as major effectors and targets of health and performance. Different kinds of stimuli and resulting homeostatic perturbations (i.e., metabolic, mechanical, neuronal, and hormonal) are reflected, taking into account their role in the local and systemic deflection of molecular sensors and mediators, and their role in tissue and organ adaptations. In skeletal muscle, a high metabolic perturbation induced by high-intensity training is the major stimulus for skeletal muscle adaptation. In the cardio-vascular system, high-intensity training induces haemodynamic stress and deflection of the $\mathrm{Ca}^{2+}$ handling as major stimuli for functional and structural adaptation of the heart and vessels. For the immune system haemodynamic stress, hormones, exosomes, and $\mathrm{O}_{2}$ availability are proposed stimuli that mediate their effects by alteration of different signalling processes leading to local and systemic (anti)inflammatory responses. Overall, high-intensity training shows specific molecular signatures that demonstrate its high potential to improve health and physical performance. 


\section{Introduction}

Physical training can improve performance and health by changes in almost all organs and tissues in the human body. In competitive sports, it is common practice to use different types of exercise protocols to achieve specific adaptation in the athlete's body to optimise physical performance. High-intensity training (HIT) and sprint-interval training (SIT) have enjoyed increasing popularity in elite sports since the middle of the last century besides high-volume training (HVT). At the beginning of this century, it has been suggested that training methods with higher intensity such as HIT and SIT can provide beneficial effects for patients with a chronic disease and should be included in the comprehensive medical management plan [1]. For an optimised use of HIT and SIT to increase physical performance and/or health, it is important to understand the molecular and cellular mechanisms of this training in the different target groups. A specific feature of HIT, and even more of SIT, is the high physical strain occurring only for a limited time. Therefore, HIT and SIT are characterised by specific metabolic, mechanic, hormonal, and neuronal perturbations in different tissue and organs with subsequent systemic, local and cellular signalling [2-4]. This integrative review aims to demonstrate molecular signatures of HIT and SIT in selected tissues and organs with respect to acute effects and chronic adaptations. The review focuses primarily on HIT or SIT, but comparisons to HVT are mentioned when available. As this manuscript is an integrative review, the literature search was not conducted systematically and, therefore, does not claim to consider all published articles in the presented research field. The included studies had to follow a mechanistic approach.

\section{Definition of Intensity Zones in Endurance Training}

In recent years, there has been a lively discussion about the potential of different endurance training protocols, especially of different training intensities, in inducing endurance-type adaptations and health benefits. One of the first studies to trigger this discussion and comparing short-term SIT with traditional continuous lowintensity endurance training (HVT) found similar adaptations after two weeks of training, despite the large difference in training volume [5]. Hundreds of publications dealing with this topic followed.

- Figure 1 gives an overview of the range of intensity of the endurance training and the training zones currently used, including its current nomenclature. Generally, HVT (Zone 1) consists of continuous exercise at $50-75 \%$ of $\mathrm{VO}_{2}$ max and durations of $1-6 \mathrm{~h} \mathrm{[6].}$ The following Zone 2 is called the "threshold zone", also mainly consisting of continuous exercise with intensities of $75-85 \%$ of $\mathrm{VO}_{2}$ max or between lactate threshold 1 (LT1) and the maximal lactate stead state (MLSS) and durations 45-90 min. There has not been a clear nomenclature and differentiation for and between the last two zones, but at present, it is mainly differentiated as HIT (Zone 3) and SIT (Zone 4). The prescription for HIT and SIT consists of the manipulation of several variables including interval intensity and duration, recovery interval intensity and duration, number of repetitions, and number of series [7]. HIT includes longer intervals of $2-5$ min and intensities of $>85-100 \%$ of $\mathrm{VO}_{2}$ max, and SIT con- sists of short bouts ( $\leq 45 \mathrm{sec}$ ) of "all-out" intervals, both interspersed with recovery periods [7]. Generally, the work:rest ratios during HIT are larger $(\geq 1)$ than during SIT $(\leq 1)$.

For simplicity, we refer to the above-mentioned classification in this review. However, it must be taken into account that the SIT zone covers a very large speed or power range compared to the other zones (i. e., HVT, Threshold, HIT). Therefore, it has been suggested that all-out training can be further divided into repeated sprint training (RST), consisting of short intervals (3-10 sec), and SIT, consisting of long all-out intervals ( $30-45 \mathrm{sec}$ ). The manipulation of training variables can lead to very different acute reactions and long-term adaptations, e.g., prolongation of duration alone with the same intensity and work:rest ratio leads to higher glycogen depletion and blood lactate levels. This illustrates that even small modifications to the protocol can change the outcome, and thus, complicate the comparison between studies.

However, according to the mentioned studies, some questions remain unanswered. For example, why these completely different training regimes can induce similar training adaptations? However, the use of a polarized training approach by elite athletes shows that a clear difference between HVT, HIT and SIT is very likely to exist [8]. Currently, it is suggested that it is necessary to perform high volumes of low-intensity training (70-80\%) to maximize peripheral adaptations and low volumes of HIT/SIT (10-20\%) to maximize cardiac adaptations $[8,9]$.

\section{Stimulus- and Perturbation-Based Exercise Prescriptions}

It will be necessary in the future to think about additional physiological stimulus-based and perturbation-based (metabolic, mechanical, neuronal and hormonal) exercise prescriptions alongside the usual ones, and to understand how these stimuli and resulting homeostatic perturbations challenge and disrupt cellular homeostasis. Of course, the numerous training parameters (i. e., intensity, volume, frequency etc.) and their different combinations during each exercise session, as well as the complex nature of the whole training regimes is far too complex to find a clear relationship between a training stimulus (i. e., a certain combination of stimuli and resulting homeostatic perturbations) and long-term adaptation. This complex processing nature of single training sessions and whole training regimes on the cellular level might be one reason why it is impossible to recommend an "ideal" training program for each individual based on sound scientific evidence [10]. However, it should be a first step to think about the general induction of stimuli and the resulting homeostatic perturbations via a specific training intervention. This might include the distribution, duration, amplitude and frequency of stimuli, and the four specific homeostatic perturbations. This train of thought has already been introduced by Laursen [11]. Based on several molecular studies about HVT, HIT and SIT, Laursen et al. suggested that HIT and SIT are more or less energy (metabolic) driven. During HIT, and especially during SIT, the cell is confronted with maximal energy demand in a short time which leads to a change in the ATP/AMP ratio and an activation of adenosine monophosphate kinase (AMPK). The AMPK is a kinase that modulates cellular metabolism acutely 


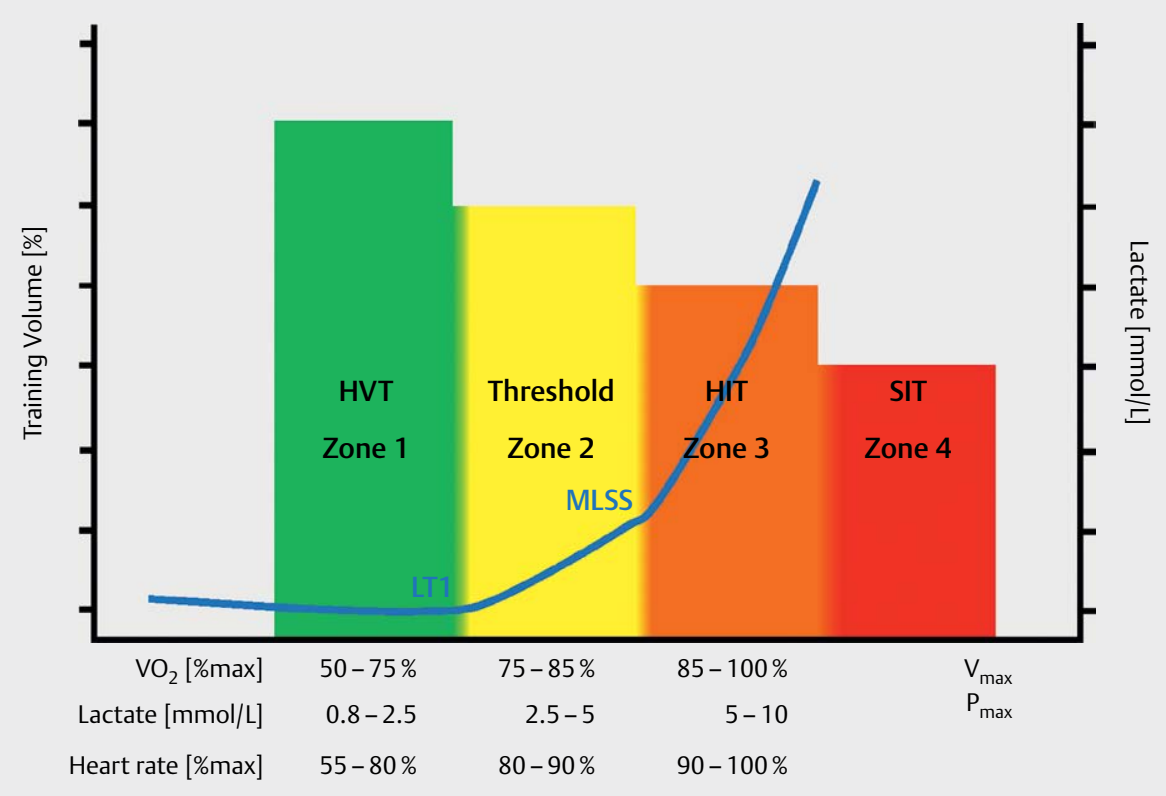

- Fig. 1 Training intensity zones used for the control of endurance training. High-volume training (HVT) is performed for $>60$ min at $50-75 \%$ of $\mathrm{VO}_{2}$ max or mainly up to lactate threshold (LT). Threshold training is performed for 45-60 min between LT and the maximal lactate steady state (MLSS). High-intensity training (HIT) is performed at longer intervals (4-5 min) and $85-100 \%$ of $\mathrm{VO}_{2}$ max. Sprint-Interval-Training (SIT) is mainly performed using all-out bouts of $\leq 30 \mathrm{sec}$. The blue line illustrates an exemplified lactate curve generated during a step test. $V_{\text {max }} / P_{\text {max }}:$ maximal velocity/power output.

through phosphorylation of metabolic enzymes and, over time, via transcriptional regulation [12]. On the other hand, HVT seems to be dominated by the activation of $\mathrm{Ca}^{2+}$ signalling pathways. The numerous sub-maximal repeated contractions during HVT are related to a constant $\mathrm{Ca}^{2+}{ }_{-}$-flux in the muscle cell and a prolonged rise in intramuscular which activates calcium-calmodulin kinase (CaMK). Both increases in AMPK and CaMK are associated with an increase in peroxisome proliferator-activated receptor- $y$ co-activator- $1 \alpha(\mathrm{PGC}-1 \alpha)$ mRNA, a transcriptional co-activator that has been described in the literature as the "master switch" for mitochondrial biogenesis. It is of note that the increase in AMPK and the following increase of PGC- $1 \alpha$ after SIT occurred without an increase in CaMK [11]. This example shows that improvements in endurance performance may be achieved through either high volumes or high intensities of endurance training, despite the activation of different molecular sensors and signalling pathways. In addition to the role of AMPK in mitochondrial biogenesis, the induction of this enzyme is implicated in regulating glucose uptake during exercise as well as exercise-induced insulin-sensitizing effects [13]. Furthermore, there is growing evidence that AMPK is increasingly involved in mitigating endoplasmic reticulum stress $[14,15]$. AMPK is further regulated by extracellular receptor-mediated stimuli such as adipokines, catecholamines, and interleukins, suggesting many roles for AMPK in normal physiology, pathophysiology, and beyond energy-sensing [16].

In addition to mitochondrial biogenesis, such stimulus-and perturbation-based exercise descriptions also need to be established for other adaptational processes, like angiogenesis. As already mentioned, changes in $\mathrm{Ca}^{2+}$, Adenosine diphosphate (ADP), Adenosine monophosphate (AMP), partial pressure of oxygen $\left(\mathrm{PO}_{2}\right)$, and shear stress activates AMPK/ CaMK-PGC-1 $\alpha$, Hypoxia-inducible factor 1-alpha (HIF-1 $\alpha$ ), and nitric oxide (NO) signalling, which leads to the up-regulation of vascular endothelial growth factor (VEGF) expression and other growth factors [17]. Therefore, also in this case, different stimuli and homeostatic perturbations seem to trigger angiogenic processes. But again, changes in $\mathrm{Ca}^{2+}, A D P$, $\mathrm{AMP}, \mathrm{PO}_{2}$, and shear stress are highly affected by the training protocol. Any exercise carried out is characterised by a specific blend of stimuli and resulting homeostatic perturbations ( $\bullet$ Fig. 2 ). The manipulation of intensity, duration, or recovery periods etc. will lead to different stimuli and resulting homeostatic perturbations [11] and, therefore, to different responses [2], or however, perhaps even similar reactions in biomarkers and adaptations [7]. It was shown that capillarization increases after moderate, as well as after HIT [18-21]. However, the extent to which the increase in capillarization occurs for the same functional purposes in response to different intensities is not known. Moderate-intensity training may induce capillary growth via $\mathrm{Ca}^{2+}$ and prolonged shear stress, to expand the oxygen diffusion capacity by improving the diffusion area, reducing diffusion distance and increasing mean transit time. High-intensity training with a large contribution of anaerobic energy production may induce capillary growth via AMPK, local hypoxia, and high shear stress in a short period of time to improve the capacity for the removal of metabolites.

\section{General Mechanisms Induced by Hit Leading to Molecular Signalling}

In recent years, there has been a fundamental change in (molecular) exercise physiology, from the rather general "supercompensation hy- 


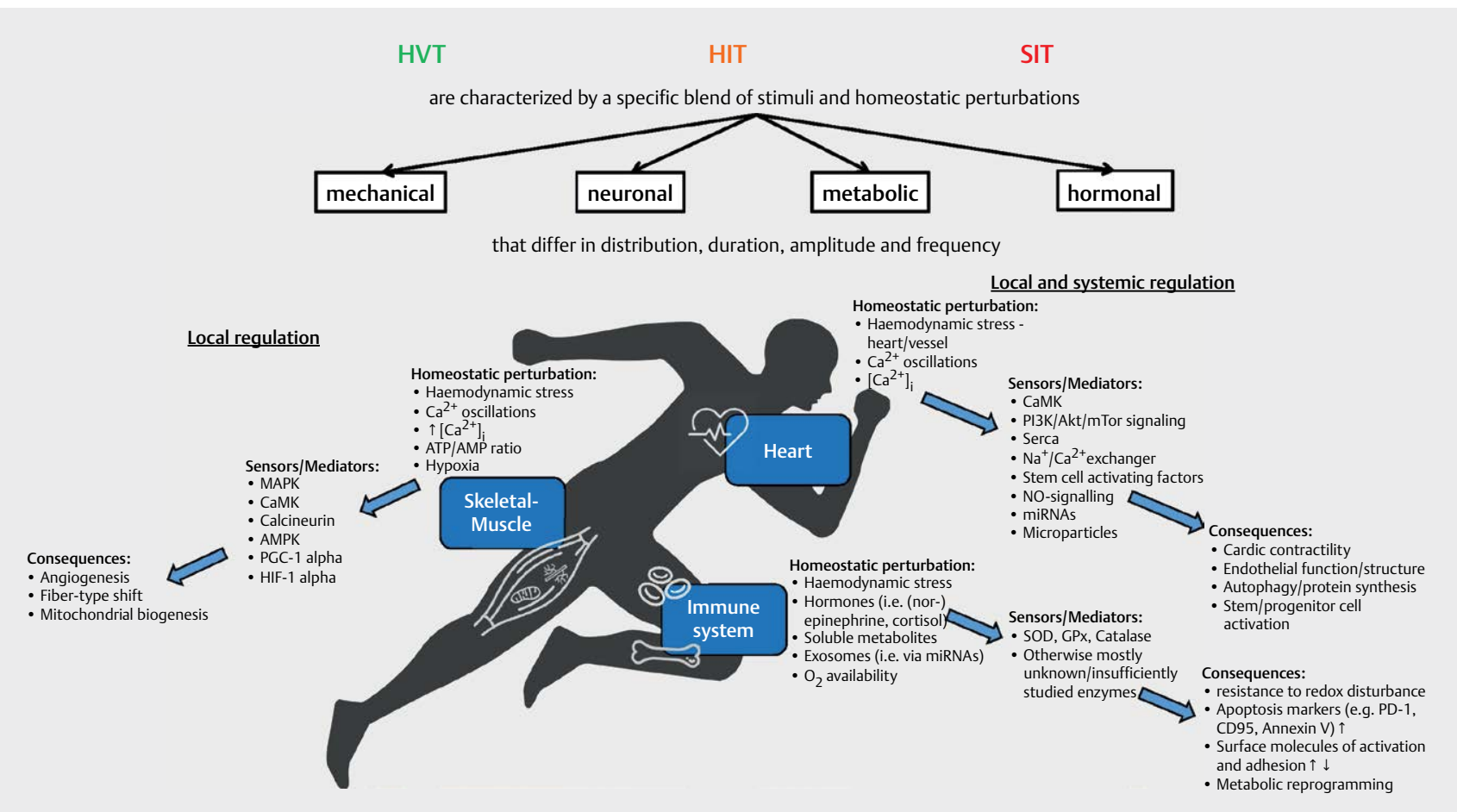

Fig. 2 Potential stressors during exercises of different intensity and volume: high-intensity training (HIT), sprint interval training (SIT), high volume training (HVT). Mechanical load, hormonal adjustments, neuronal activation and metabolic disturbances all induce specific signalling events, transcription/translation, finally leading to adaptations.

pothesis" to the more specific "signal-transduction-hypothesis" of adaptation. According to this "signal-transduction-hypothesis", specific sensor proteins detect environmental or exercise-related signals, which are then further processed by transduction/signalling pathways, finally leading to changes in gene transcription, gene translation, protein synthesis or breakdown [2]. This results in the adaptation of the cell, the organ or the whole organism. In this way, exercise-related molecular signals can either be sensed at the whole organism level (i. e., endocrine and nervous system) or by organs, specific tissues, and individual cells containing the necessary machinery to sense changes in their local environment [10].

Any exercise carried out is characterised by a specific blend of stimuli and resulting homeostatic perturbations, to which tissues (e. g., muscle, endothelium) are subjected when activated. Hoppeler [2] and other researchers distinguished (1) mechanical load, (2) hormonal adjustment, (3) neuronal activation, and (4) metabolic disturbance as the main identifiable homeostatic perturbations. Mechanical load includes tension, strain, pressure, and stretch. Hormonal adjustments include acute and long-term changes in systemic or local (i. e., autocrine, paracrine) hormone levels, as well as changes in hormone receptors. Metabolic disturbances are induced by chemical changes on the systemic, tissue or cellular level, including the energy status (i. e., ATP/AMP-ratio, free fatty acids [FFA], glycogen), hypoxia, $\mathrm{H}^{+}$-ions, lactate, $\mathrm{P}_{\mathrm{i}}$ and reactive oxygen species (ROS). Neuronal activation serves to integrate the duration, amplitude and frequency of intracellular $\mathrm{Ca}^{2+}$ fluctuations [2]. Even though all four perturbations are induced by all kinds of exercise above a certain-often not easy to define-level, some perturbations dominate in certain types of exercise. In the so-called strength training situation, we typically find mechanical load and hormonal adjustments to be the dominant homeostatic perturbations. In the so-called endurance training, the mechanical load is low and metabolic disturbances, neuronal activation, and hormonal adjustments are high and usually persist over longer time periods [2,22]. All these signals have a dual purpose. They serve to re-establish the homeostasis disrupted by exercise, however, they also serve to modify tissue so as to make it more efficient when dealing with similar homeostatic perturbations in the future (adaptation) [2]. An acute (signalling) response and signalling related to long-term adaptations of tissues must therefore be distinguished.

In order to obtain a better understanding of adaptation processes and to recommend effective and specific training programs/ schedules, it is absolutely necessary to identify what kind of exercise regimen induces different kind and amount of stimuli and homeostatic perturbations, what kind of tissues and cells are activated and what causal connection exists between the cellular response and the training adaptations. The magnitude and type of adaptation strongly depend on the specific molecular and cellular response, which is governed by the training stimulus and the individual prerequisites (genome and epigenome). In order to assign acute reactions and long-term adaptations to specific training, the external training load needs to be described and characterised as accurately as possible, and potential stimuli need to be identified. 


\section{Impact of Hit Regimes on Different Organ Systems}

\section{Muscle}

Studies directly comparing HVT with HIT or SIT often use exercise protocols that are matched for total-work or energy-expenditure (isocaloric). Although these types of matching are useful for (certain) research questions or special populations (e. g., untrained subjects, patients), we must keep in mind that they are less meaningful for athletes training, as the different types of protocols differ much more in volume in elite sports. Furthermore, the molecular response to acute exercise might differ significantly depending on the training status. In trained muscles signalling pathways and gene expression might be activated to a lesser extent after exercise than in untrained muscles [23].

To investigate the effects of different exercise protocols on skeletal muscle, acute exercise studies measure changes (i. e., phosphorylation, gene expression, or protein synthesis) in a variety of signalling proteins such as AMPK, CaMK, MAPK, and PGC- $1 \alpha$ ( Fig. 2). PGC- $1 \alpha$, a transcriptional coactivator, plays an important role in the regulation and coordination of mitochondrial biogenesis in skeletal muscle by controlling its oxidative enzyme expression [24]. The most important exercise-related regulators of PGC- $1 \alpha$ are p38 MAPK, CaMKs, and AMPK. These signalling molecules induce different $P G C-1 \alpha$ post-translational modifications (i.e., phosphorylation, acetylation, methylation, and ubiquitination) that regulate its stability and ability to interact with transcription factors $[25,26]$. Activated PGC- $1 \alpha$ might translocate from the cytoplasm to the nucleus $[27,28]$ and mitochondria [29], where it modulates the transcriptional activity of its molecular partners and thus regulates gene expression.

As the ATP turnover rate during exercise can be over 100 -fold greater than at rest [12], and AMPK activity dynamics are determined principally by ADP concentration [30], AMPK activity increases in response to cellular energy demand or exercise intensity, respectively. Effects of directbinding activators depend on their concentrations, binding affinities for AMPK, abilities to enhance AMPK phosphorylation, and the magnitudes of their allosteric activation of AMPK [30]. Calcium plays an important role during muscle contraction and allows the interaction between actin and myosin. After an action potential, $\mathrm{Ca}^{2+}$ is released from the sarcoplasmic reticulum and is transported back into the SR by Sarcoplasmic/endoplasmic reticulum calcium ATPase (SERCA) afterwards. The amplitude and duration of $\mathrm{Ca}^{2+}$-oscillations depend on the level of force output by the muscle and thus on the intensity of the exercise, respectively. These oscillations modulate the kinase activity of CaMK, mainly CaMKII, which is the dominant isoform in skeletal muscle. Thereby, CaMKII is suggested to act as a stimulation-frequency decoder, being sensitive to different types of contractions based on the amplitude and frequency of calcium transients. This can result in different signalling transduction patterns [12]. p38 mitogen-activated protein kinase (p38MAPK) can stimulate upstream transcription factors of the PGC- $1 \alpha$ gene. Constitutive activation of p38 MAPK increases markers of mitochondrial adaptation in skeletal muscle.

Egan et al. [31] compared the effects of two isocaloric (1674 kJ) bouts of continuous cycling performed at either $40 \%$ or $80 \%$ of peak oxygen consumption ( $\mathrm{VO}_{2}$ peak) on skeletal muscle signalling.
PGC-1 $\alpha$ mRNA increased 3.8-fold after low-intensity exercise, whereas it increased 10.2-fold after HIT. This generally supports an intensity-dependent regulation of PGC- $1 \alpha$ expression. Also, Di Donato et al. [32] were able to show that mitochondrial protein synthesis was significantly greater after a more intense (30 min at $60 \%$ $W_{\text {max }}$ ) compared to a work-matched low exercise trial (60 min at $\left.30 \% \mathrm{~W}_{\text {max }}\right)$.

Besides the intensity itself, the interval character (on-off-pattern of HIT or SIT) might be important too. The comparison of two work matched protocols $([\sim 317 \mathrm{~kJ}]$ a continuous [30-min] and an intermittent exercise [ $30 \times 1$-min intervals separated by 1 -min of recovery] at $70 \%$ of $\mathrm{VO}_{2}$ peak) revealed that AMPK, CaMKII, and p38-MAPK phosphorylation was greater when the session of the moderate-intensity exercise was divided into 1 min intervals, interspersed with rest, compared to when it was performed as a continuous 30 min session [33]. However, some studies failed to demonstrate acute differences between intermittent and continuous exercise. Two different running protocols (HIT: $6 \times 3$-min at $90 \%$ $\mathrm{VO}_{2}$ max interspersed with 3-min recovery periods at $50 \% \mathrm{VO}_{2}$ max vs. HVT: 50 -min continuous running at $70 \% \mathrm{VO}_{2}$ max) matched for average intensity, duration, and distance induced similar activation p38MAPK and PGC-1 mRNA transcription [34]. Also, four $30 \mathrm{sec}$ Wingate tests interspersed with 4 min of rest (SIT) or a bout of continuous exercise that was matched for total work $(67 \pm 7 \mathrm{~kJ})$ elicited similar increases in AMPK and p38 MAPK, as well as PGC-1 $\alpha$ mRNA expression [35]. However, despite similar acute signalling responses to the continuous and SIT protocols, continuous training did not increase the maximal activity or protein content of several mitochondrial markers.

Despite the mentioned and other reported increases in PGC- $1 \alpha$ mRNA expression after SIT [36], protein content often remained unchanged. These same findings were also shown for HVT lasting either $1 \mathrm{~h}$ [37] or $3 \mathrm{~h}$ [38]. Concerning the PGC-1 $\alpha$ protein, several other changes have been described. The study of Burgomaster et al. 2008 [39] suggests that more than one bout of SIT (6 weeks; 18 sessions) is necessary to increase PGC- $1 \alpha$ protein content. However, after a reduced number of sessions ( 2 weeks; 6 sessions; $8-12 \times 60$-sec intervals at $100 \%$ of peak power output elicited during a ramp $\mathrm{VO}_{2}$ peak test), total PGC- $1 \alpha$ protein content remained unchanged. Though, the nuclear abundance of PGC- $1 \alpha$ was $25 \%$ higher after training [40], suggesting a rapid upregulation of mitochondrial gene transcription without the need for increases in PGC- $1 \alpha$ total protein content. Burgomaster et al. also suggested that an increase in nuclear PGC- $1 \alpha$ may be a more dominant mechanism of regulation in the trained state when PGC- $1 \alpha$ total protein is already upregulated [39].

Another factor that generally has to be considered is the involvement of muscle mass or the recruitment of different muscle fibre types. Their recruitment during exercise depends on both intensity and duration: type I fibres are mainly recruited at low-intensity exercise ( $<40 \%$ of $\mathrm{VO}_{2}$ peak) while increasing intensity leads to greater type II fibre recruitment [12]. When muscles are stimulated at physiological frequencies, intracellular $\mathrm{Ca}^{2+}$ concentration reaches 100-300 nM in slow-twitch (type I) fibres but may reach concentrations that are 10 -fold higher $(1-2 \mu \mathrm{M})$ in fast-twitch fibres [41]. Additionally, the increase in recruitment, which corresponds to the increase in the exercise intensity, leads to a progressive ac- 
cumulation of various metabolites (e. g., ADP) and the development of fatigue in a working muscle. This might be one reason why AMPK and CaMKII are more activated after HIT/SIT involving more type II fibres. Indeed, skeletal muscle AMPK phosphorylation and AMPK expression are fibre type-specific in humans in the basal state, as well as during exercise [42].

Phosphorylation of AMPK $\alpha 1 / 2$ Thr172 and several specific AMPK targets in type II fibres were more pronounced after HIT $\left(6 \times 1.5 \mathrm{~min}, 95 \% \mathrm{VO}_{2} \mathrm{max}+2.5 \mathrm{~min}, 40 \% \mathrm{VO}_{2} \mathrm{max}\right)$, while in type I fibres, these parameters changed to the same extent after both HIT and HVT (30 min, 69\% VO 2 max) [43]. Consequently, PGC-1 expression was 2.2-and 6-fold greater in type Ila than in type I and IIx fibres after 6 weeks of interval training consisting of 5 to 6 intervals of $1-3$ min at $70-80 \%$ of $\mathrm{VO}_{2}$ peak [44].

\section{Heart and blood-vessels}

Improvement of the cardiovascular system to enhance the physical performance capacity by HIT is not limited to elite and recreational sports. HIT is also known to improve the performance and health of organs and tissues, including the cardiovascular system, in terms of preventive health and rehabilitation. Further, HIT exerts its beneficial effects mainly by high metabolic and mechanic perturbations and subsequent hormonal and neuronal deflection via different molecular mechanisms targeting the heart and vessels to change function and structure as shown in human and animal models ( $\triangleright$ Fig. 2). Therefore, molecular and cellular mechanisms are described leading to acute and chronic adaptation of the heart and the vascular system.

HIT has been shown to improve cardiac function and structure through activation of $\mathrm{Ca}^{2+} / \mathrm{CaMKII}$, leading to hyperphosphorylation of phospholamban [45] with subsequent activation of SERCA2a. In conjunction with an increased expression of the $\mathrm{Na}+1$ $\mathrm{Ca}^{2+}$ exchanger [46], this leads to improved $\mathrm{Ca}^{2+}$ cycling and finally to better cardiomyocyte contractility. $\mathrm{Ca}^{2+}$ handling can also be restored to normal levels as a result of raised transverse tubule ( $\mathrm{T}$ tubule) density, sarcoplasmic reticulum synchrony of $\mathrm{Ca}^{2+}$ release, and sarcoplasmic reticulum $\mathrm{Ca}^{2+}$-ATPase (SERCA2a; $\mathrm{Ca}^{2+}$ transporter) activity [47].

Physiological hypertrophy of the heart can be induced by the phosphoinositol-3 kinase/Akt/ mammalian target of rapamycin (mTOR) signal transduction pathway that leads to higher ribosomal biogenesis and protein synthesis [48]. HIT can also induce cardiac hypertrophy by alteration of autophagy-related gene expression such as LC3 and p62, mTOR [49]. HIT can also increase cardioprotection and decrease heart injuries through the increase in G-CSF, G-CSFR, and C-kit levels which are known as stem cell recruitment factors [50]. HIT improved cardiac function and levels of stem cell and cardiomyocyte markers, and reduced infarct size. The levels of stem cell regulating factors such as GATA4, Nkx2.5, and c-Kit, Sca-1, and CITED4 were significantly increased by a different kind of exercise, while the expression of the stem cell silencing factor $C / E B P \beta$ is reduced in the rat exercise postinfarction model. The effect is more pronounced by HIT [51].

Furthermore, incremental changes in the $\mathrm{NO}-, \mathrm{NO}_{3}{ }^{-}, \mathrm{NO}_{2}{ }^{-}$axis may be one of the mechanisms through which a HIT program can contribute to myocardial protection and decrease myocardial infarct size after ischemia/reperfusion injury [52]. Although HIT is also able to reverse cardiac remodelling in the diabetic heart, it seems unable to restore the nitroso-redox imbalance imposed and observed in this condition [53].

In the vascular system, the effect of HIT can be explained by intracellular and extracellular/systemic signalling leading to communication in and between tissue and organ. Besides other molecular factors, miRNAs and microparticles have the potential to spread molecular signalling throughout the body via the vascular system. miRNAs (such as microRNAs -16, -21 , and -126) attenuate endothelial inflammation and may mediate vascular-protective effects of physical exercise including HIT [54, 55]. HIT leads also to decreased endothelial microparticles (EMP) in the blood and promotes a phosphatidylserine-dependent uptake of EMP into target endothelial cells, which is associated with the protection of target cells against apoptosis. The EMPs are carriers of various molecules, such as growth factors and miRNA, which show their biological relevance as signal transducers [56].

Most of the effects of exercise on the vascular endothelium are mediated by intermittent increases of laminar shear stress. On the luminal side of the endothelial cells, direct signalling can occur through deformation of VEGF receptor 2 (VEGFR2) or platelet endothelial cell adhesion molecule 1 (PECAM1) activating phosphatidylinositol 3-kinase (PI3K) to phosphorylate Akt (PKB) and induce Akt mediated endothelial NO synthase (eNOS) phosphorylation, leading to higher NO production. PDK, phosphoinositide-dependent kinase [57]. The shear stress-mediated regulation of endothelial cells is dependent on endothelial glycocalyx. Therefore, it seems interesting that 4 weeks of HIT induces a structural alteration of the microvascular glycocalyx. It has been demonstrated that microvascular glycocalyx thickness is dependent on physical performance level and glycocalyx thickness increased with the improvement in exercise capacity after HIT. The increase in glycocalyx thickness was predicted by an acute elevation of miRNA-143 levels [58]. In an animal model, it was shown that mechanisms altering endothelial function such as increased expression of MMP2 and 9 as well as increased protein modification by advanced glycosylation end products (AGE) can be counterbalanced by HIT [59].

A further structural effect of HIT is related to the microvascular system. HIT can increase the capillary density by an increase of endothelial proliferation in a VEGF and bFGF independent manner in skeletal muscle [20]. The improvement of capillarization could be explained by the stimulation of other pro-angiogenic factors (such as SDF-1 and MMPs) that enhances the mobilisation/functionality of endothelial progenitor cells. Furthermore, the vascular resistance against hypoxia can be increased by that way, as well as the depressed shedding of vascular endothelial cells induced by HIT [60]. HIT increases circulating endothelial progenitor cells (EPC), promotes the migration and tube formation of EPCs, diminishes the shedding of endothelial cells, and elevates plasma nitrite plus nitrate, stromal cell-derived factor-1, MMP-9, and vascular endothelial growth factor-A concentrations enhancing EPC functionality and suppressing endothelial injury [60]. Altogether, HIT induces several functional and structural alterations involved in the maintenance of the cardiovascular system by intracellular signalling and extracellular molecular carrier allowing communication in the whole vascular system and the related tissue and organs. 


\section{Immune system}

An active lifestyle that includes regular exercise is supposed to reduce the risk of non-communicable inflammatory diseases by enhancing the function of the immune system and by gradually reestablishing immune homeostasis $[61,62]$. Similar effects account for exercise-induced health benefits in several disease populations. HIT represents an exercise regimen that is increasingly investigated over the last decade in both innate and adaptive immune cell subsets. In this regard, training intensity seems to have a selective effect on T cell subset apoptosis and mobilization into circulation. Krüger et al. [63] observed a greater mobilization and apoptotic response in highly differentiated $T$ cells of healthy untrained males immediately after HIT exercise, whereas moderate continuous training (here referred to as $\mathrm{HVT}$ ) at $70 \% \mathrm{VO}_{2 \max }$ mainly affected apoptosis in low differentiated T cells, including regulatory $\mathrm{CD}^{+}{ }^{+}$regulatory $\mathrm{T}$ cells, in the recovery period ( $3 \mathrm{~h}$ post-exercise). The authors suggest that the subsetspecific pattern of T cell mobilization and apoptosis might influence infection immunology and the inflammatory response. Recently, Wadley et al. [64] showed that both acute HIT $\left(10 \times 4\right.$-min intervals at $85 \% \mathrm{VO}_{2 \max }$, 2 min rest between intervals) and $\mathrm{HVT}\left(60 \% \mathrm{VO}_{2 \max }\right)$ are able to increase the number of circulating programmed cell death receptors (PD-1)-positive $C D 8^{+} T$ cells immediately after exercise. $\mathrm{PD}-1$ is a membrane-bound receptor that functions as an immune checkpoint to reduce cell-mediated immunity by increasing apoptosis of antigen-experienced effector $T$ cells [65]. Interestingly, only HIT increased the cell count of PD- $1^{+}$central memory $C D 8^{+} T$ cells. Although representing only a small component of the exercise-induced lymphocytosis, the authors suggest that the HITinduced increase of this PD- $1^{+} \mathrm{CD} 8^{+} \mathrm{T}$ cell subset may be a homeostatic (anti-inflammatory) mechanism to limit T cell receptor activation, differentiation, and redistribution after exercise. Another recent study revealed a decrease in the mean fluorescence intensity of surface PD- 1 in the overall CD8 ${ }^{+} T$ cell subset $1 \mathrm{~h}$ after $\mathrm{HVT}$ at $60 \%$ peak power output [66]. Despite differences in sample size, the time point of significance, and the level of cell phenotyping, an intensity-dependent effect can be assumed but needs further validation.

It is suggested that a beneficial effect of exercise on the cellular level is linked to an upregulation of antioxidants and damage repair enzymes in response to an exercise-induced increase in oxidative stress and consecutive damage of intracellular lipid and protein molecules [67] ( $\triangleright$ Fig. 2). It is supposed that these cellular alterations are intensity-dependent. If the homeostatic perturbation is above-threshold, exercise may induce apoptosis in subsets of both the innate and adaptive immune system through diverse pathways (e. g., increase in FasR expression and cytosolic cytochrome c level, decrease in the mitochondrial membrane potential $\left(\Delta \Psi_{\mathrm{m}}\right)$ and $\mathrm{B}$ cell lymphoma 2 (Bcl-2) level, lipid peroxidation) [68]. How these pathways, which determine cell fate, are linked to redox signalling is currently a hot topic in the field of immunometabolism [69]. By investigating the effect of HIT $(4 \times 30$-sec sprints, 4 min rest between intervals) and $\mathrm{HVT}\left(70 \% \mathrm{VO}_{2 \max }\right)$ on leukocytosis and redox status in plasma [70], it was shown that HIT led to a significant increase in leukocytes, protein carbonyls, and total antioxidant capacity immediately post-exercise compared to HVT. To reveal changes on the cellular level, Fisher et al. [71] examined the effect of three HIT sessions, each separated by $48 \mathrm{~h}$, on antioxidant enzyme (i. e., superoxide dismutase, catalase, glutathione peroxidase) gene expression and activity in the lymphocyte compartment. Although no differences in antioxidant gene expression were ob- served at any time point both within (pre, post, $3 \mathrm{~h}$ post, $24 \mathrm{~h}$ post) and between days (day one to three), the activity of superoxide dismutase (all training days), catalase (day one and two) and glutathione peroxidase (day one and three) were significantly increased immediately post-exercise. This suggests an exercise-induced disturbance in cellular redox status which may persist for some time, as the lymphocyte viability (assessed by in vitro $\mathrm{H}_{2} \mathrm{O}_{2}$ exposure) was decreased at $3 \mathrm{~h}$ post-exercise compared to viability levels at pre and $24 \mathrm{~h}$ post-exercise. Importantly, an adaption to exercise-induced oxidative stress on the cellular level can be assumed as lymphocyte viability at $3 \mathrm{~h}$ post-exercise increased on days two and three compared to day one.

Considering alterations of gene products and functional capacity in neutrophils as the most abundant cell type of the human immune system (50-70\% of all leukocytes) in response to HIT, Ottone et al. [72] identified increased levels of both superoxide dismutase and reduced glutathione as potent antioxidative molecules $24 \mathrm{~h}$ after a HIT session. Furthermore, functional analysis revealed enhanced function (phagocytic activity, generation of reactive species) at $24 \mathrm{~h}$ post-exercise. Similar results were yielded when healthy adults conducted HIT ( 15 to 60 -sec intervals at $>90 \%$ $\mathrm{HR}_{\max }, 45-120$-sec rest between intervals [for 18-20 min]) or HVT $\left(70 \% \mathrm{HR}_{\max }\right)$ thrice weekly over ten weeks [73]. In addition to an increased phagocytic capacity, neutrophils showed an enhanced oxidative burst (NADPH-oxidase dependent reactive oxygen species generation) when co-cultured with Escherichia coli. The same observations were made in monocytes that represent another important cellular compartment of the innate immune system. Interestingly, both exercise regimes altered monocyte, but not neutrophil, expression of key functional surface receptors (i. e., TLR4, TLR2, and CD16) which suggests that the increased functional capacity of neutrophils is a consequence of altered intracellular signalling. Of note, a single HIT session was enough to decrease TLR2 expression on classical and $\mathrm{CD}_{16}{ }^{+}$monocytes, but not neutrophils, in diabetic and healthy adults [74], thereby potentially suppressing inflammatory signalling. No effect was seen in TLR4 expression in any cell population which suggests that, at least for monocytes, repetitive exercise is necessary to decrease TLR4 levels. This is supported by Robinson et al. [75] who showed that ten sessions of HIT over two weeks decreased the expression of TLR4 in monocytes of prediabetic people. However, no reductions were observed for TLR2 expression, whereas a decrease of both TLR2 and TLR4 was shown in lymphocytes. Differences in the staining strategy need to be taken into account when interpreting results (see $>$ Table 1 ).

Recent findings show significant reductions of the neutrophil-tolymphocyte ratio (NLR) after three weeks of HIT compared to HVT in multiple sclerosis patients [76]. Another study in elite athletes showed that HIT $(4 \times 4$ min at $90-95 \%$ PPO $)$ and SIT $(4 \times 30$ sec all-out $)$ increased both the NLR and neutrophil counts three hours post-exercise, with higher deltas being evident for the NLR in the SIT group [77]. These findings indicate an intensity-dependent and cohort-independent effect of exercise on neutrophils which needs to be further elucidated by methods measuring intracellular signalling.

Although there is an increasing appreciation for HIT as an effective exercise regime to beneficially affect the immune system, a sufficient amount of data is lacking to draw clear conclusions about cellular alterations in response to HIT ( $\bullet$ Fig. 2). To serve the huge 
- Table 1 Differential expression of pattern recognition receptors on immune cells after High-intensity training.

\begin{tabular}{|c|c|c|c|c|}
\hline Author & HIIT Protocol & Population & Cell type \& Staining & PRR \\
\hline $\begin{array}{l}\text { Bartlett } \\
2017[73]\end{array}$ & $\begin{array}{l}\text { Chronic exercise ( } 10 \text { weeks, } 3 x / \\
\text { week), cycling, } 18-25 \text { min HIIT with } \\
\text { bouts of } 15-60 \text { sec at } 90 \% \mathrm{HR}_{\max }\end{array}$ & $\begin{array}{l}\text { healthy inactive } \\
\text { subjects, } 43 \pm 11 \text { yrs }\end{array}$ & $\begin{array}{l}\mathrm{CD}^{+} 6^{+} \text {Neutrophils } \\
\text { Monocytes } \\
\text { Classical (CD14 }{ }^{+} \text {CD16-) } \\
\left.\text { Intermediate (CD14 }{ }^{+} \text {CD16 } 6^{\text {int }}\right) \\
\left.\text { Non-classical (CD14 }{ }^{+} \text {CD } 16^{\text {bight }}\right) \\
\end{array}$ & $\begin{array}{l}\text { TLR2 } \leftrightarrow \text { TLR4 } \leftrightarrow \\
\text { TLR2 } \leftrightarrow \text { TLR4 } \leftrightarrow \\
\text { TLR2 } \downarrow \text { TLR4 } \leftrightarrow \\
\text { TLR2 } \leftrightarrow \text { TLR4 } \downarrow\end{array}$ \\
\hline $\begin{array}{l}\text { Bartlett } \\
2018[83]\end{array}$ & $\begin{array}{l}\text { Chronic exercise ( } 10 \text { weeks, } 3 \times \text { / } \\
\text { week), treadmill, } 30 \text { min HIIT with } \\
\text { bouts of } 60-90 \text { sec at a } \mathrm{HR} \\
\text { corresponding to } 80-90 \% \text { of } \mathrm{VO}_{2} \\
\text { reserve }\end{array}$ & $\begin{array}{l}\text { inactive subjects, RA, } \\
64 \pm 7 \mathrm{yrs}\end{array}$ & 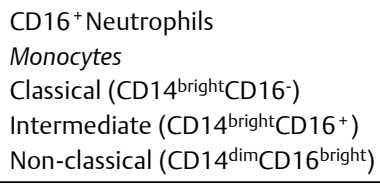 & $\begin{array}{l}\text { TLR4 } \\
\text { TLR2 } \leftrightarrow \text { TLR4 } \leftrightarrow \\
\text { TLR2 } \downarrow \text { TLR4 } \downarrow \\
\text { TLR2 } \leftrightarrow \text { TLR4 } \leftrightarrow \\
\end{array}$ \\
\hline $\begin{array}{l}\text { Robinson } \\
2015 \text { [75] }\end{array}$ & $\begin{array}{l}\text { Chronic exercise ( } 2 \text { weeks, } 10 \\
\text { progressive sessions), cycling, } \\
4-10 \times 60 \text { sec at } 85-90 \% W_{\text {peak }}\end{array}$ & $\begin{array}{l}\text { Prediabetic inactive } \\
\text { subjects, } 52 \pm 10 \mathrm{yrs}\end{array}$ & $\begin{array}{l}\text { CD15 }{ }^{+} \text {Neutrophils } \\
\text { CD } 14^{+} \text {Monocytes } \\
\text { Lymphocytes }\end{array}$ & $\begin{array}{l}\text { TLR2 } \leftrightarrow \text { TLR4 } \downarrow \\
\text { TLR2 } \leftrightarrow \text { TLR4 } \downarrow \\
\text { TLR2 } \downarrow \text { TLR4 } \downarrow \\
\end{array}$ \\
\hline $\begin{array}{l}\text { Durrer } \\
2017 \text { [74] }\end{array}$ & $\begin{array}{l}\text { Acute exercise, cycling, } 7 \times 60 \mathrm{sec} \text { at } \\
85 \% \text { peak power output }\end{array}$ & $\begin{array}{l}\mathrm{T} 2 \mathrm{D}+\text { healthy } \\
\text { controls, }>50 \mathrm{yrs}\end{array}$ & $\begin{array}{l}\text { CD16 }{ }^{+} \text {Neutrophils } \\
\text { Monocytes } \\
\text { Classical }\left(\mathrm{CD} 14^{+} \mathrm{CD} 16^{-}\right) \\
\text {Non-classical }\left(\mathrm{CD} 14^{+} \mathrm{CD} 16^{+}\right)\end{array}$ & $\begin{array}{l}\text { TLR2 } \leftrightarrow \text { TLR4 } \leftrightarrow \\
\text { TLR2 } \downarrow \text { TLR4 } \leftrightarrow \\
\text { TLR2 } \downarrow \text { TLR4 } \leftrightarrow \\
\text { All changes account for } \\
\text { immediately post and } 1 \mathrm{~h} \\
\text { post }\end{array}$ \\
\hline
\end{tabular}

$\mathrm{HR}_{(\max )}:$ (maximal) Heart rate; PRR: Pattern Recognition Receptors; RA: Rheumatoid arthritis; TLR: Toll-like receptor; T2D: Type 2 Diabetes mellitus; yrs: years.

heterogeneity and phenotypic complexity within each immune cell subset, in-depth analyses that consider precise phenotyping, cellular metabolism, and cell signalling, also to the DNA level, are highly recommended. Functional assays should be included wherever possible to determine clinically relevant changes. This is particularly important when it comes to optimising exercise interventions in a disease context-dependent manner where specific immune subsets play major roles (i. e., NK cells and CD8 ${ }^{+} T$ cells in cancer or regulatory $\mathrm{CD} 4^{+} \mathrm{T}$ cells in autoimmunity).

\section{Summary and Concluding Remarks}

Studies have shown that exercise intensity is an important stimulus. The manipulation of exercise variables, in this case, the intensity within interval training, changes the demands, stimuli and homeostatic perturbations induced, and affects the acute and chronic molecular response. Therefore, the mode of interval training should be carefully planned according to the main goal of the training session or the exercise intervention.

This requires exact quantification of the external training load, as well as quantification of the internal training load, in the short and long term. The aim to screen adaptational processes might require the combined investigation of intercellular (systemic) and intracellular signalling events between or in well-known affected tissues such as the muscle, the heart, or the different components of the immune system. A major challenge for future studies will be to gain a more profound knowledge of training interventions, the metabolic, mechanical, hormonal and neuronal stimuli induced, the subsequent local and systemic signalling and their effects on the adaptive cellular response in skeletal muscle, vascular system and heart. Considering the immune system, it is important to increase the knowledge of intensity-dependent metabolic and functional changes in adaptive and innate immune cells, respectively.
Although disturbances in both compartments are linked to physical inactivity (i. e., main drivers of chronic inflammatory diseases), there is a lack of sufficient HIT-based data from humans studies to conclude a "re-establishment" of immune homeostasis on the tissue and systemic level [78]. Therefore, the implementation of HIT/ SIT into the daily training process or the general periodization is an important question.

In addition to the aspects discussed in this review regarding the molecular signature of HIT and SIT, redox biology is increasingly recognized as an indispensable variable involved in a variety of stress and adaptation responses in different cell types, and thus directly associated with "healthy" and "diseased" states $[69,79,80]$. Since HIT induces significant metabolic, mechanical, and hormonal homeostatic perturbations in various tissues, exercise-induced (intracellular) redox reactions and associated signalling may be of major interest when investigating tissue-specific exercise responses and adaptations. However, literature on HIT/SIT-induced changes in redox signalling is increasing but still sparse [81, 82], thereby underscoring the need to put research in redox biology into the spotlight in the next years.

\section{Main Findings}

- Any exercise carried out is characterised by a specific blend of stimuli and resulting homeostatic perturbations.

- AMPK activity and PGC-1 $\alpha$ expression increase in response to cellular energy demand or exercise intensity, respectively.

- The increased recruitment of type II fibres, as a result of increasing exercise intensity, could be one reason why AMPK and CaMKII are more activated after HIT/SIT.

- HIT induces distinct metabolic and mechanical as well as hormonal and neuronal perturbations resulting in functional and structural adaptations of heart and vessel. 
- Different local and systemic sensors/mediators are involved in cellular, subcellular and extracellular modulation of the heart and vessels.

- Molecular signalling and cellular responses following HIT are largely unknown in components of the innate and adaptive immune system.

- The main question to be answered: How do acute HIT-induced changes in the molecular landscape of immune cells affect their phenotype and functionality in the long term?

\section{Acknowledgements}

This research was conducted in the absence of any external funding. We thank Christine Koliamitra and Florian Javelle for language editing.

\section{Conflict of Interest}

The authors declare that they have no conflict of interest.

\section{References}

[1] Ross LM, Porter RR, Durstine JL. High-intensity interval training (HIIT) for patients with chronic diseases. J Sport Health Sci 2016; 5: 139-144

[2] Hoppeler H, Baum O, Lurman G et al. Molecular mechanisms of muscle plasticity with exercise. Compr Physiol 2011; 1: 1383-1412

[3] Ben-Zeev T, Okun E. High-intensity functional training: Molecular mechanisms and benefits. Neuromolecular Med 2021; 3: 335-338

[4] Jiménez-Maldonado A, García-Suárez PC, Rentería I et al. Impact of high-intensity interval training and sprint interval training on peripheral markers of glycemic control in metabolic syndrome and type 2 diabetes. Biochim Biophys Acta Mol Basis Dis 2020; 1866: 165820

[5] Gibala M], Little JP, van Essen M et al. Short-term sprint interval versus traditional endurance training: Similar initial adaptations in human skeletal muscle and exercise performance. J Physiol 2006; 575: 901-911

[6] Seiler S, Tønnessen E. Intervals, thresholds, and long slow distance: The role of intensity and duration in endurance training. Sportscience 2009; 13: 32-53

[7] Buchheit M, Laursen PB. High-intensity interval training, solutions to the programming puzzle: Part I: Cardiopulmonary emphasis. Sports Med 2013; 43: 313-338

[8] Yu H, Chen X, Zhu W et al. A quasi-experimental study of Chinese top-level speed skaters' training load: Threshold versus polarized model. Int J Sports Physiol Perform 2012; 7: 103-112

[9] Stöggl T, Sperlich B. Polarized training has greater impact on key endurance variables than threshold, high intensity, or high volume training. Front Physiol 2014; 5: 33

[10] Wackerhage H. Molecules ageing and exercise. In: Wackerhage H. (ed) Muscular Exercise Physiology: An Introduction. London: Routledge; 2014

[11] Laursen PB. Training for intense exercise performance: High-intensity or high-volume training? Scand J Med Sci Sports 2010; 20: 1-10

[12] Egan B, Zierath JR. Exercise metabolism and the molecular regulation of skeletal muscle adaptation. Cell Metab 2013; 17: 162-184

[13] O'Neill HM. AMPK and exercise: Glucose uptake and insulin sensitivity. Diabetes Metab J 2013; 37: 1-21
[14] Bae HR, Kim DH, Park MH et al. $\beta$-Hydroxybutyrate suppresses inflammasome formation by ameliorating endoplasmic reticulum stress via AMPK activation. Oncotarget 2016; 7: 66444-66454

[15] Liu JQ, Zhang L, Yao J et al. AMPK alleviates endoplasmic reticulum stress by inducing the ER-chaperone ORP150 via FOXO1 to protect human bronchial cells from apoptosis. Biochem Biophys Res Commun 2018; 497: 564-570

[16] Dasgupta B, Chhipa RR. Evolving lessons on the complex role of ampk in normal physiology and cancer. Trends Pharmacol Sci 2016; 37: 192-206

[17] Arany Z, Foo S-Y, Ma Y et al. HIF-independent regulation of VEGF and angiogenesis by the transcriptional coactivator PGC-1alpha. Nature 2008; 451: 1008-1012

[18] Gavin TP, Ruster RS, Carrithers JA et al. No difference in the skeletal muscle angiogenic response to aerobic exercise training between young and aged men. J Physiol 2007; 585: 231-239

[19] Hoier B, Nordsborg N, Andersen S et al. Pro-and anti-angiogenic factors in human skeletal muscle in response to acute exercise and training. J Physiol 2012; 590: 595-606

[20] Jensen L, Bangsbo J, Hellsten Y. Effect of high intensity training on capillarization and presence of angiogenic factors in human skeletal muscle. J Physiol 2004; 557: 571-582

[21] Klausen K, Andersen LB, Pelle I. Adaptive changes in work capacity, skeletal muscle capillarization and enzyme levels during training and detraining. Acta Physiol Scand 1981; 113: 9-16

[22] Wackerhage H. Molecular Exercise Physiology: An Introduction. London: Routledge; 2014

[23] Popov DV. Adaptation of skeletal muscles to contractile activity of varying duration and intensity: The role of PGC- $1 \alpha$. Biochemistry (Mosc) 2018; 83: 613-628

[24] Gibala M. Molecular responses to high-intensity interval exercise. Appl Physiol Nutr Metab 2009; 34: 428-432

[25] Kupr B, Handschin C. Complex coordination of cell plasticity by a PGC-1 $\alpha$-controlled transcriptional network in skeletal muscle. Front Physiol 2015; 6: 325

[26] Lira VA, Benton CR, Yan Z et al. PGC-1alpha regulation by exercise training and its influences on muscle function and insulin sensitivity. Am J Physiol Endocrinol Metab 2010; 299: E145-E161

[27] Little JP, Safdar A, Cermak N et al. Acute endurance exercise increases the nuclear abundance of PGC-1alpha in trained human skeletal muscle. Am J Physiol Regul Integr Comp Physiol 2010; 298: R912R917

[28] Wright DC, Han D-H, Garcia-Roves PM et al. Exercise-induced mitochondrial biogenesis begins before the increase in muscle PGC-1alpha expression. J Biol Chem 2007; 282: 194-199

[29] Smith BK, Mukai K, Lally JS et al. AMP-activated protein kinase is required for exercise-induced peroxisome proliferator-activated receptor co-activator 1 translocation to subsarcolemmal mitochondria in skeletal muscle. J Physiol 2013; 591: 1551-1561

[30] Coccimiglio IF, Clarke DC. ADP is the dominant controller of AMP-activated protein kinase activity dynamics in skeletal muscle during exercise. PLoS Comput Biol 2020; 16: e1008079

[31] Egan B, Carson BP, Garcia-Roves PM et al. Exercise intensity-dependent regulation of peroxisome proliferator-activated receptor coactivator-1 mRNA abundance is associated with differential activation of upstream signalling kinases in human skeletal muscle. J Physiol 2010; 588: 1779-1790

[32] Di Donato DM, West DWD, Churchward-Venne TA et al. Influence of aerobic exercise intensity on myofibrillar and mitochondrial protein synthesis in young men during early and late postexercise recovery. Am J Physiol Endocrinol Metab 2014; 306: E1025-E1032 
[33] Combes A, Dekerle J, Webborn $\mathrm{N}$ et al. Exercise-induced metabolic fluctuations influence AMPK, p38-MAPK and CaMKII phosphorylation in human skeletal muscle. Physiol Rep 2015; 3: e12462

[34] Bartlett JD, Hwa Joo C, Jeong T-S et al. Matched work high-intensity interval and continuous running induce similar increases in PGC- $1 \alpha$ mRNA, AMPK, p38, and $\mathrm{p} 53$ phosphorylation in human skeletal muscle. J Appl Physiol (1985) 2012; 112: 1135-1143

[35] Cochran AJR, Percival ME, Tricarico S et al. Intermittent and continuous high-intensity exercise training induce similar acute but different chronic muscle adaptations. Exp Physiol 2014; 99: 782-791

[36] Gibala MJ, McGee SL, Garnham AP et al. Brief intense interval exercise activates AMPK and p38 MAPK signaling and increases the expression of PGC-1alpha in human skeletal muscle. J Appl Physiol (1985) 2009; 106: 929-934

[37] Coffey VG, Zhong Z, Shield A et al. Early signaling responses to divergent exercise stimuli in skeletal muscle from well-trained humans. FASEB J 2006; 20: 190-192

[38] Watt MJ, Southgate RJ, Holmes AG et al. Suppression of plasma free fatty acids upregulates peroxisome proliferator-activated receptor (PPAR) alpha and delta and PPAR coactivator 1alpha in human skeletal muscle, but not lipid regulatory genes. J Mol Endocrinol 2004; 33: 533-544

[39] Burgomaster KA, Howarth KR, Phillips SM et al. Similar metabolic adaptations during exercise after low volume sprint interval and traditional endurance training in humans. J Physiol 2008; 586: $151-160$

[40] Little JP, Safdar A, Wilkin GP et al. A practical model of low-volume high-intensity interval training induces mitochondrial biogenesis in human skeletal muscle: Potential mechanisms. J Physiol 2010; 588: 1011-1022

[41] Hennig R, Lømo T. Firing patterns of motor units in normal rats. Nature 1985; 314: 164-166

[42] Lee-Young RS, Canny B], Myers DE et al. AMPK activation is fiber type specific in human skeletal muscle: Effects of exercise and short-term exercise training. J Appl Physiol (1985) 2009; 107: 283-289

[43] Kristensen DE, Albers PH, Prats C et al. Human muscle fibre typespecific regulation of AMPK and downstream targets by exercise. J Physiol 2015; 593: 2053-2069

[44] Russell AP, Feilchenfeldt J, Schreiber $S$ et al. Endurance training in humans leads to fiber type-specific increases in levels of peroxisome proliferator-activated receptor-gamma coactivator- 1 and peroxisome proliferator-activated receptor-alpha in skeletal muscle. Diabetes 2003; 52: 2874-2881

[45] Kemi O], Ellingsen $\varnothing$, Ceci $M$ et al. Aerobic interval training enhances cardiomyocyte contractility and $\mathrm{Ca}^{2}+$ cycling by phosphorylation of CaMKII and Thr-17 of phospholamban. J Mol Cell Cardiol 2007; 43: 354-361

[46] Wisløff U, Loennechen JP, Falck G et al. Increased contractility and calcium sensitivity in cardiac myocytes isolated from endurance trained rats. Cardiovasc Res 2001; 50: 495-508

[47] Stølen TO, Høydal MA, Kemi O] et al. Interval training normalizes cardiomyocyte function, diastolic $\mathrm{Ca}^{2}+$ control, and SR $\mathrm{Ca}^{2}+$ release synchronicity In a mouse model of diabetic cardiomyopathy. Circ Res 2009; 105: 527-536

[48] Cassidy S, Thoma C, Houghton D et al. High-intensity interval training: A review of its impact on glucose control and cardiometabolic health. Diabetologia 2017; 60: 7-23

[49] Gunadi JW, Tarawan VM, Setiawan I et al. Cardiac hypertrophy is stimulated by altered training intensity and correlates with autophagy modulation in male Wistar rats. BMC Sports Sci Med Rehabil 2019; 11: 9
[50] Ghanimati R, Rajabi H, Ramezani F et al. The effect of preconditioning with high-intensity training on tissue levels of G-CSF, its receptor and $\mathrm{C}$-kit after an acute myocardial infarction in male rats. BMC Cardiovasc Disord 2020; 20: 75

[51] Naderi N, Hemmatinafar M, Gaeini AA et al. High-intensity interval training increase GATA4, CITED4 and c-Kit and decreases C/EBP $\beta$ in rats after myocardial infarction. Life Sci 2019; 221: 319-326

[52] Fallahi A, Gaeini A, Shekarfroush S et al. Cardioprotective effect of high intensity interval training and nitric oxide metabolites $\left(\mathrm{NO}^{-}, \mathrm{NO}^{-}{ }^{-}\right.$). Iran J Public Health 2015; 44: 1270-1276

[53] Novoa U, Arauna D, Moran M et al. High-intensity exercise reduces cardiac fibrosis and hypertrophy but does not restore the nitrosoredox imbalance in diabetic cardiomyopathy. Oxid Med Cell Longev 2017; 2017: 7921363

[54] Schmitz B, Breulmann FL, Jubran B et al. A three-step approach identifies novel shear stress-sensitive endothelial microRNAs involved in vasculoprotective effects of high-intensity interval training (HIIT). Oncotarget 2019; 10: 3625-3640

[55] Wahl P, Wehmeier UF, Jansen FJ et al. Acute effects of different exercise protocols on the circulating vascular microRNAs -16, -21, and -126 in trained subjects. Front Physiol 2016; 7: 643

[56] Wahl P, Jansen F, Achtzehn S et al. Effects of high intensity training and high volume training on endothelial microparticles and angiogenic growth factors. PLoS One 2014; 9: e96024

[57] Adams V, Reich B, Uhlemann M et al. Molecular effects of exercise training in patients with cardiovascular disease: Focus on skeletal muscle, endothelium, and myocardium. Am J Physiol Heart Circ Physiol 2017; 313: H72-H88

[58] Schmitz B, Niehues $H$, Lenders $M$ et al. Effects of high-intensity interval training on microvascular glycocalyx and associated microRNAs. Am J Physiol Heart Circ Physiol 2019; 316: H1538-H1551

[59] Adams V, Alves M, Fischer T et al. High-intensity interval training attenuates endothelial dysfunction in a Dahl salt-sensitive rat model of heart failure with preserved ejection fraction. J Appl Physiol (1985) 2015; 119: 745-752

[60] Tsai HH, Lin CP, Lin YH et al. High-intensity Interval training enhances mobilization/functionality of endothelial progenitor cells and depressed shedding of vascular endothelial cells undergoing hypoxia. Eur J Appl Physiol 2016; 116: 2375-2388

[61] Neufer PD, Bamman MM, Muoio DM et al. understanding the cellular and molecular mechanisms of physical activity-induced health benefits. Cell Metab 2015; 22: 4-11

[62] Walsh NP, Gleeson MM, Shephard RJ et al. Institutional Repository Position statement part one : Immune function and exercise. Exerc Immunol Rev 2011; 17: 6-63

[63] Krüger K, Alack K, Ringseis R et al. Apoptosis of T-Cell subsets after acute high-intensity interval exercise. Med Sci Sports Exerc 2016; 48: 2021-2029

[64] Wadley A], Cullen T, Vautrinot J et al. High intensity interval exercise increases the frequency of peripheral PD $-1+\mathrm{CD} 8{ }^{+}$central memory T-cells and soluble PD-L1 in humans. Brain Behav Immun Health 2020; 3: 100049

[65] Postow MA, Callahan MK, Wolchok JD. Immune checkpoint blockade in cancer therapy. J Clin Oncol 2015; 33: 1974-1982

[66] Schenk A, Joisten N, Walzik D et al. Acute exercise impacts AhR and PD-1 levels of CD8 ${ }^{+} \mathrm{T}$-cells-Exploratory results from a randomized cross-over trial comparing endurance versus resistance exercise. Eur J Appl Physiol 2020; 121: 637-644

[67] Radak Z, Ishihara K, Tekus E et al. Exercise, oxidants, and antioxidants change the shape of the bell-shaped hormesis curve. Redox Biol 2017; 12: $285-290$ 
[68] Krüger K, Mooren FC. Exercise-induced leukocyte apoptosis. Exerc Immunol Rev 2014; 20: 117-134

[69] Muri ], Kopf M. Redox regulation of immunometabolism. Nat Rev Immunol 2020; 21: 363-381

[70] Jamurtas AZ, Fatouros IG, Deli CK et al. The effects of acute low-volume HIIT and aerobic exercise on leukocyte count and redox status. J Sports Sci Med 2018; 17: 501-508

[71] Fisher G, Schwartz DD, Quindry J et al. Lymphocyte enzymatic antioxidant responses to oxidative stress following high-intensity interval exercise. J Appl Physiol (1985) 2011; 110: 730-737

[72] De Oliveira Ottone V, Costa KB, Tossige-Gomes R et al. Late neutrophil priming following a single session of high-intensity interval exercise. Int J Sports Med 2019; 40: 171-179

[73] Bartlett DB, Shepherd SO, Wilson OJ et al. Neutrophil and monocyte bactericidal responses to 10 weeks of low-volume high-intensity interval or moderate-intensity continuous training in sedentary adults. Oxid Med Cell Longev 2017; 2017: 8148742

[74] Durrer C, Francois M, Neudorf $\mathrm{H}$ et al. Acute high-intensity interval exercise reduces human monocyte toll-like receptor 2 expression in type 2 diabetes. Am J Physiol Regul Integr Comp Physiol 2017; 312: R529-R538

[75] Robinson E, Durrer C, Simtchouk S et al. Short-term high-intensity interval and moderate-intensity continuous training reduce leukocyte TLR4 in inactive adults at elevated risk of type 2 diabetes. J Appl Physiol (1985) 2015; 119: 508-516
[76] Joisten N, Proschinger S, Rademacher A et al. High-intensity interval training reduces neutrophil-to-lymphocyte ratio in persons with multiple sclerosis during inpatient rehabilitation. Mult Scler 2021; 27: 1136-1139

[77] Wahl P, Mathes S, Bloch W et al. Acute impact of recovery on the restoration of cellular immunological homeostasis. Int J Sports Med 2020; 41: 12-20

[78] Furman D, Campisi J, Verdin E et al. Chronic inflammation in the etiology of disease across the life span. Nat Med 2019; 25: 1822-1832

[79] Horn A, Van der Meulen JH, Defour A et al. Mitochondrial redox signaling enables repair of injured skeletal muscle cells. Sci Signal 2017; 10: eaaj1978

[80] Schieber M, Chandel NS. ROS function in redox signaling and oxidative stress. Curr Biol 2014; 24: 453-462

[81] Louzada RA, Bouviere J, Matta LP et al. Redox signaling in widespread health benefits of exercise. Antioxid Redox Signal 2020. Online ahead of print. doi: 10.1089/ars.2019.7949

[82] Parker L, Stepto NK, Shaw CS et al. Acute high-intensity interval exercise-induced redox signaling is associated with enhanced insulin sensitivity in obese middle-aged men. Front Physiol 2016; 7: 411

[83] Bartlett DB, Willis LH, Slentz CA et al. Ten weeks of high-intensity interval walk training is associated with reduced disease activity and improved innate immune function in older adults with rheumatoid arthritis: A pilot study. Arthritis Res Ther 2018; 20: 127 